\title{
Vaginal cuff dehiscence after vaginal cuff brachytherapy for uterine cancer. A case report
}

\author{
Richard Cattaneo II, MD, Maria Bellon, MS, Mohamed A. Elshaikh, MD \\ Department of Radiation Oncology. Henry Ford Hospital, Detroit, MI, USA
}

\begin{abstract}
Vaginal cuff dehiscence is a rare, but potentially serious complication after total hysterectomy. We report a case of vaginal cuff dehiscence after vaginal cuff brachytherapy. A 62 year old female underwent a robotic-assisted laparoscopic hysterectomy with bilateral salpingo-oophorectomy, and was found to have International Federation of Gynecology and Obstetrics (FIGO) 2009 stage IB endometrioid adenocarcinoma of the uterus. The patient was referred for adjuvant vaginal cuff brachytherapy. During the radiation treatment simulation, a computerized tomography (CT) of the pelvis showed abnormal position of the vaginal cylinder. She was found to have vaginal cuff dehiscence that required immediate surgical repair. Vaginal cuff dehiscence triggered by vaginal cuff brachytherapy is very rare with only one case report in the literature.
\end{abstract}

Key words: uterine carcinoma, brachytherapy, hysterectomy, vaginal dehiscence.

\section{Purpose}

Vaginal cuff dehiscence (VCD) is a rare complication after hysterectomy. The reported incidence of VCD following abdominal hysterectomy is less than one percent [1]. With the increased use of laparoscopic, including robotic-assisted approaches, there appears to be an increased rate of VCD. The rate of this complication is relatively higher after roboticassisted hysterectomy reaching up to $4.1 \%$ [2].

Predisposing factors for VCD include poor wound healing, excessive pressure at the vaginal vault e.g. sexual intercourse [3,4], and vaginal instrumentation [5]. The use of adjuvant vaginal cuff brachytherapy for patients with endometrial cancer is effective in reducing vaginal vault recurrence [6], and there has been increased utilization of this adjuvant treatment in the United States [7]. We report a case of vaginal cuff dehiscence after vaginal cuff brachytherapy with a review of relevant literature. Although VCD is still very rare after vaginal cuff brachytherapy, the patients should be counseled about this rare treatment-related complication.

\section{Case report}

In April 2012, a 62 year old Caucasian postmenopausal female elected to undergo robotic-assisted laparoscopic hysterectomy with bilateral salpingo-oophorectomy for her complex endometrial hyperplasia without atypia. Final pathology revealed FIGO 2009 stage IB endometrioid adenocarcinoma, FIGO grade 1 with invasion of $6 \mathrm{~mm}$ of the $10 \mathrm{~mm}$ thick myometrium. After discussion of her case in Gynecologic Oncology multidisciplinary conference, she was recommended to receive adjuvant vaginal cuff brachytherapy. At consultation, she denied pain, vaginal bleeding or vaginal discharge. She presented for CT simulation for vaginal brachytherapy treatment planning approximately 9 weeks after her hysterectomy. During simulation, the vaginal cylinder was noted to project abnormally high into the pelvis (Fig. 1). While there was no vaginal bleeding or discharge with the insertion of the vaginal cylinder, there was a small amount of blood on the vaginal cylinder upon removal. The patient's gynecologic oncologist was contacted, and the patient was evaluated immediately. On speculum examination, there was evidence of complete vaginal cuff dehiscence with healthy appearing small bowel noted at the vaginal cuff opening without other abnormalities appreciated. On the same day, the patient underwent surgical closure of vaginal cuff via trans-vaginal approach using four sutures with \#0 Vicryl in figure-of-eight fashion. She was discharged home the following day in stable condition.

\section{Discussion}

Vaginal cuff dehiscence is a known, but unusual complication after hysterectomy. Reported rates for VCD after trans-abdominal hysterectomy have been reported as low as $0.03 \%$, and are generally less than $1 \%[1,8]$. However, with the increased use of laparoscopic, including robotic-assisted approaches due to a variety of benefits intra-operatively and post-operatively, there has been an increase in the rate of VCD. Rates of VCD with robotic hysterectomy have been reported as $0.98 \%$ from the University of Pennsylvania experience [9], and $4.1 \%$ from a retrospective review from 

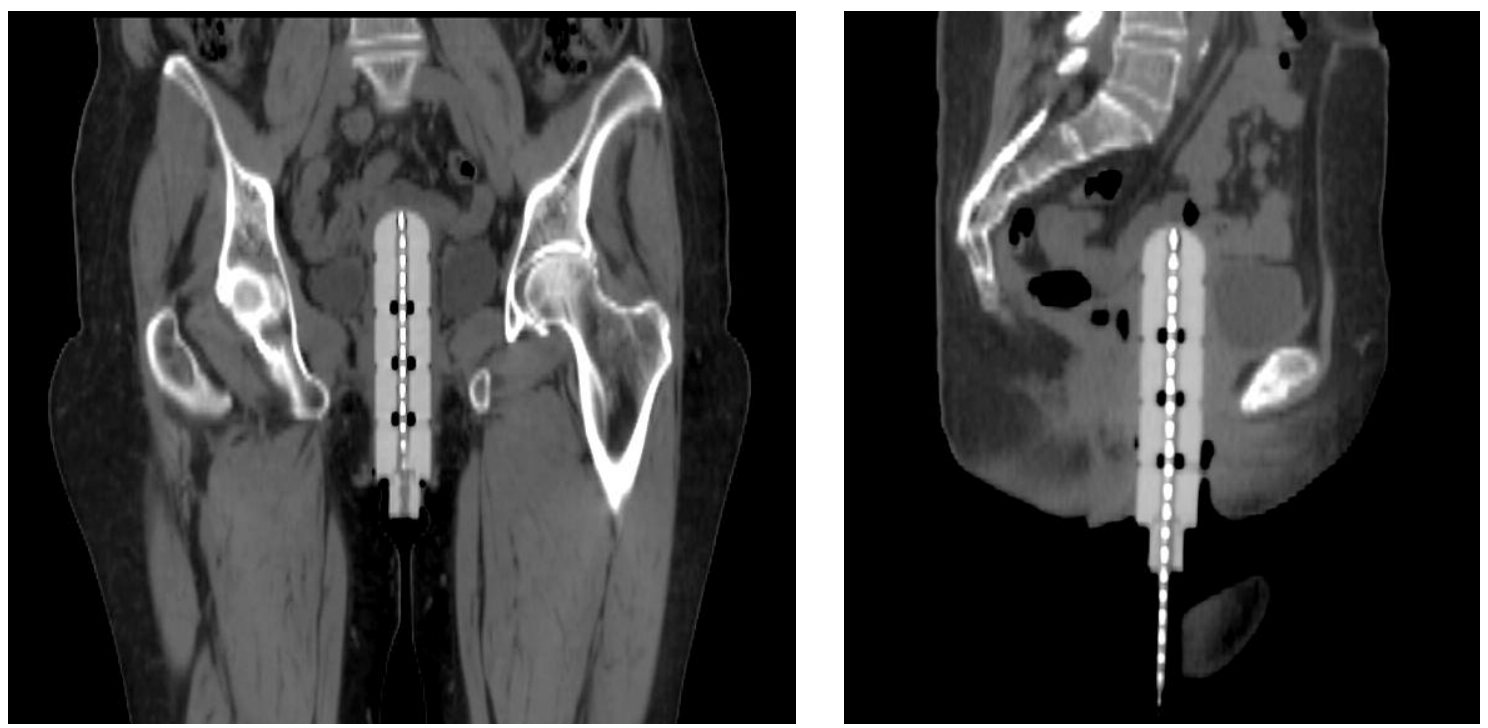

Fig. 1. Vaginal cylinder located abnormally in the pelvis

Mayo Clinic in Scottsdale, AZ [2]. There are a variety of theories on the reason for this increase; including the use of electrocautery for colpotomy, the suturing technique used, and the magnification view causing suture bites to be smaller than desired.

Proposed risk factors for VCD include poor surgical technique, smoking, use of Valsalva maneuver, post-operative infection, hematoma, adjuvant radiation therapy, steroid use, connective tissue disease, vaginal trauma/rape, use of vaginal dilator, diabetes mellitus, chronic constipation [2,10-12]. While the precipitating event may be known, as in the case of early resumption of sexual intercourse, often it is unknown. Typically, the cause of VCD has been dependent on menopause status. Vaginal cuff dehiscence in post-menopausal patients has been associated with chronic pelvic prolapse or having a short, atrophic vagina [1]. In premenopausal patients, VCD has been typically associated with coitus prior to vaginal cuff healing [10]. The timing of this rare complication is very important. While most of it occurs in the first three months after hysterectomy $[13,14]$, some patients develop VCD more than four months after hysterectomy [4].

There is a recent case report of two patients that experienced VCD after intracavitary vaginal HDR brachytherapy treatment [5]. Our patient is a post-menopausal, former smoker that underwent robotic-assisted laparoscopic hysterectomy approximately nine weeks prior to vaginal brachytherapy. She otherwise did not have other risk factors for VCD. She did not complain of any vaginal discharge or vaginal bleeding. She was noted to have a small amount of blood on the vaginal cylinder after CT simulation, and the cylinder was noted to project high on her images. She was then referred for evaluation and surgical correction by her gynecologic oncologist. There is debate in the literature concerning the best management of VCD, but early corrective intervention is necessary [8]. The radiation oncologist should be aware of the very low risk of VCD, and take steps to aide in early detection and prevention of this rare complication. This very rare complication can be prevent- ed by initiating vaginal brachytherapy no sooner than six weeks after hysterectomy to allow adequate healing time [15]. However, delaying the start of adjuvant radiation treatment more than 9 weeks after hysterectomy may be associated with increased risk of tumor recurrence [16] with worse survival endpoints in patients with uterine cancer [17].

At the time of the brachytherapy simulation, careful speculum and manual examination of the vaginal cuff for findings of VCD can be useful. The location of the vaginal cylinder should be radiologically verified with each application to rule out abnormal positioning of the cylinder. Vaginal cuff deshiscence should be ruled out if the vaginal cylinder projects deep into the pelvis. To reduce the risk of dehiscence during insertion of the vaginal cylinder, it is helpful to avoid excessive pressure on the vaginal cuff. After the procedure, one should inspect the vaginal cylinder for any abnormal blood or clear vaginal fluid that could suggest dehiscence.

\section{Conclusions}

Vaginal cuff dehiscence is an unusual complication of hysterectomy. In preparation for vaginal cuff brachytherapy, the radiation oncologist should be aware of this very rare and under reported complication of vaginal brachytherapy. Most of the time, VCD can be prevented by initiating vaginal brachytherapy no sooner than six weeks after hysterectomy. However, delaying the start of vaginal cuff brachytherapy more than 9 weeks after hysterectomy may be associated with increased risk of tumor recurrence in patients with uterine cancer.

\section{References}

1. Croak A, Gebhart J, Klingele C et al. Characteristics of patients with vaginal rupture and evisceration. Obstet Gynecol 2004; 103: 572-576.

2. Kho R, Akl M, Cornella J et al. Incidence and characteristics of patients with vaginal cuff dehiscence after robotic procedures. Obstet Gynecol 2009; 114: 231-235. 
3. Kalu E, Fakokunde A, Evans-Jones J. Postcoital vault dehiscence and instestinal prolapse following abdominal hysterectomy. J Obstet Gynaecol 2006; 26: 74-75.

4. Nguyen ML, Kapoor M, Pradhan TS et al. Two cases of postcoital vaginal cuff dehiscence with small bowel evisceration after robotic-assisted laparoscopic hysterectomy. Int J Surg Case Rep 2013;4: 603-605.

5. Albuquerque K, Shah K and Potkul R. Vaginal cuff dehiscence after intracavitary brachytherapy for endometrial cancer. J Contemp Brachytherapy 2012; 4: 111-112.

6. Nout R, Smit V, Putter H et al. Vaginal brachytherapy versus pelvic external beam radiotherapy for patients with endometrial cancer of high-intermediate risk (PORTEC-2): an openlabel, non-inferiority, randomised trial. Lancet 2010; 375: 816-823.

7. Patel M, Cote M, Ali-Fehmi R et al. Trends in the utilization of adjuvant vaginal cuff brachytherapy and/or external beam radiation treatment in stage I and II endometrial cancer: a surveillance, epidemiology, and end-results study. Int J Radiat Oncol Biol Phys 2012; 83: 178-184.

8. Cronin B, Sung V, Matteson K. Vaginal cuff dehiscence: risk factors and management. Am J Obstet Gynecol 2012; 206: 284-288.

9. Robinson B, Liao J, Adams S et al. Vaginal cuff dehiscence after robotic total laparoscopic hysterectomy. Obstet Gynecol 2009; 114: 369-371.

10. Agdi M, Al-Ghafri W, Antolin R et al. Vaginal vault dehiscence after hysterectomy. J Minim Invasive Gynecol 2009; 16: 313-317.

11. Ceccaroni M, Beretta R, Malzoni M et al. Vaginal cuff dehiscence after hysterectomy: a multicenter retrospective study. Eur J Obstet Gynecol Reprod Biol 2011; 158: 308-313.

12. Somkuti S, Vieta P, Daugherty J et al. Transvaginal evisceration after hysterectomy in premenopausal women: a presentation of three cases. Am J Obstet Gynecol 1994; 171: 567-568.

13. Ceccaroni M, Berretta R, Malzoni M et al. Vaginal cuff dehiscence after hysterectomy: a multicenter retrospective study. Eur J Obstet Gynecol Reprod Biol 2011; 158: 308-313.

14. Gujar NN, Choudhari RK, Choudhari GR et al. Coitus induced vaginal evisceration in a premenopausal woman: a case report. Pat Safety Surg 2011; 5: 6.

15. Kirsner R, Eaglstein W. The wound healing process. Dermatol Clin 1993; 11: 629-640.

16. Fabrini MG, Gadducci A, Perrone F et al. Relationship between interval from surgery to radiotherapy and local recurrence rate in patients with endometrioid-type endometrial cancer: a retrospective mono-institutional Italian study. Anticancer Res 2012; 32: 169-173.

17. Elshaikh MA, Cattaneo II R, Jacobson G et al. Interval between Hysterectomy and Start of Radiation Treatment is Predictive of Recurrence in patients with endometrial carcinoma. A single Institution experience. Int J Radiat Oncol Biol Physics 2012; 84 (3 suppl.): S97. 